\title{
MULTIFOCAL IMAGE PROCESSING
}

\author{
DALIBOR MARTIŠEK AND HANA DRUCKMÜLLEROVÁ
}

\begin{abstract}
In this paper, we present a processing method for digital images from an optical microscope. High-pass type filters are generally used for image focusing. They enhance the high spatial frequencies. These filters are not appropriate if the lack of sharpness is caused by other factors. On the other hand, the (un)sharpness can be taken as an advantage and can be used for studies of the spatial distribution of structures in the observed scene. In many cases, it is possible to construct a threedimensional model of the observed object by analyzing image sharpness. Interesting two-dimensional images and a three-dimensional model can be obtained by applying the theory for multifocal image processing described in this paper. We improve the quality of the results compared to the previous methods using the Fourier transform for the analysis of local sharpness in the images.
\end{abstract}

\section{INTRODUCTION}

Three-dimensional reconstructions of object surfaces play an important role in many branches. As an example, morphological analysis of fracture surfaces reveals information on mechanical properties of construction materials (see [4-6]). The confocal microscope is a standard tool for imaging three-dimensional surfaces, however, after some post-processing using mathematical tools described in this paper, an optical microscope can be used as well.

In our paper, we will deal with partially focused images. High-pass type filters are generally used for image sharpening. They enhance the high spatial frequencies. They are, however, efficient only in cases when the picture is not sharp because of the low contrast on high frequencies (for example in a TV picture). These filters, are not appropriate if the lack of sharpness is caused by other factors such as the object being out of focus. In many cases, it is possible to construct a three-dimensional model of the observed object using more images with different focusing, as described below.

The current state of knowledge is summarized in Section 2. A broader discussion of the properties of projection in optical microscopes crucial for multifocal imaging can be found in Section 3.1, the notion of the multifocal image is introduced in Section 3.2, followed with Sections 3.3 and 3.4 that are devoted to the 2D and 3D processing with some improvements proposed in this paper.

$M S C$ (2010): primary 62H35; secondary 42B10.

Keywords: multifocal image, 3-D reconstruction, focussing criteria, Fourier transform.

This work was supported by the Grant Agency of the Czech Republic, grant no. 13-03403S. Publication of the results was financially supported by the project CZ.1.07/2.3.00/35.0004 "Popularization of BUT R\&D results and support systematic collaboration with Czech students". 


\section{Current state of Knowledge}

\subsection{Terminology for images and image sensors}

In computer graphics, data are stored at discrete points. Points are viewed as nondimensional objects conforming to the traditional Euclidean geometry. However, the display surface of an output device is a physical object and cannot display dimensionless points. For this reason, the notion of a "pixel" rather than point is used, standing for the smallest viewable formation.

In mathematical modeling, the output device is thought of as a set of isolated Euclidean points. Therefore, a color image is modeled as the vector function $f(i, j): M \rightarrow R$ where $M=\{0,1, \ldots, w-1\} \times\{0,1, \ldots, h-1\}, R \subset \mathbb{R}^{3}$, i.e., $f(i, j)=(r(i, j), g(i, j), b(i, j))$. Functions $r, g, b$ are called the color components of image $f$. Numbers $w$ and $h$ are called the image width and image height respectively. The set $M$ is called the logical plane, its elements are called logical pixels and the value $f(i, j)$ of function $f$ in pixel $[i, j]$ is called the pixel value.

An image taken with a digital camera usually has square sensors arranged in a rectangular grid, let $x, y$ be the size of its cells. We assume an image to take values at discrete points only, keeping in mind that the pixel values originated from integrals of the intensity of the light recorded over rectangle areas. We can assume that the pixel value $f(i, j)$ is integrated over

$$
P(i, j)=\left[x i-\frac{1}{2} x, x i+\frac{1}{2} x\right) \times\left[y j-\frac{1}{2} y, y j+\frac{1}{2} y\right) .
$$

The set $P(i, j)$ is called the physical pixel and the set $P$ of all physical pixels is called the physical plane.

This idea is applicable to conventional optical devices such as microscopes, too.

\subsection{Current methods and $2 \mathrm{D}$ and $3 \mathrm{D}$ reconstruction}

For an image of an object to be sharp, the object must be placed exactly in the plane to which the microscope or camera is focused [7], [8]. This plane is called a sharpness plane. However, in many cases, we cannot take the observed object to be a plane, and therefore this condition cannot be fulfilled. The sharpness plane intersects the 3-D object in a contour line. The points of this contour line will be displayed with maximum possible sharpness. Due to the wave properties of light and due to the finite resolution of the output device the image can be considered as sharp not only on this contour line, but also in a certain interval of height, the zone of sharpness. The image of the zone of sharpness is called the optical cut. In Figure 1, we can see a fracture surface of cement paste at different focal planes where the focused parts of the image are well discernible. The data for this image as well as the data for images in Figures 2 and 10 are courtesy of Prof. Tomáš Ficker (Faculty of Civil Engineering, Brno University of Technology).

To create a sharp 2D image, it is necessary to obtain a series of images of the same object, each of them with different focusing, and each point on the object focused in at least one of the images. The optical cuts are identified and the sharp parts are composed in a new image. There is also a simple method for constructing a rough $3 \mathrm{D}$ model of the object, where all points belonging to the same optical cut 

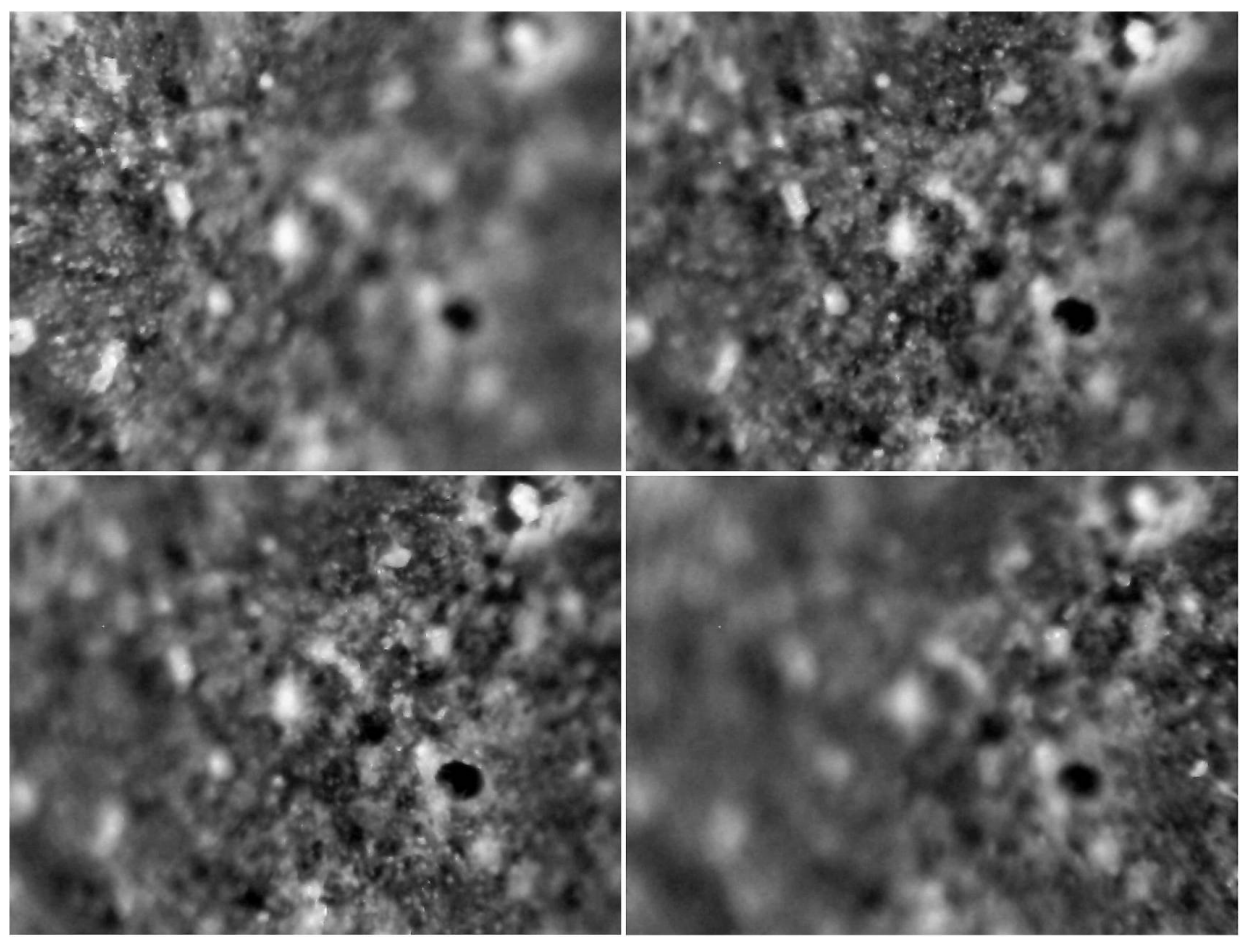

Figure 1. Different optical cuts of a 3D object (fracture surface of cement paste). Four images out of twelve were are displayed.

have the same height - the height of the corresponding zone of sharpness. This method of reconstruction is called the Method of Constant High Cuts.

Nowadays, sharp images of 3D objects and their 3D models can be obtained by means of confocal microscopes. These instruments do not show points out of the sharpness zone due to their construction and, therefore, no software identification (briefly described above) of optical cuts is necessary. The pixel height and thus also the optical cut to which the pixel belongs are identified by the hardware. Figure 2 shows a 3D reconstruction of the specimen from Figure 1 from a confocal microscope constructed from twelve optical cuts.

\section{New methods of 2D processing And 3D ReConstruction}

To achieve better results than those of Section 2.2, it is necessary to analyze the properties and limits of optical instruments. The first attempt was published in [11]. Statistical tools such as the variance and range of pixel values were used for the analysis of image sharpness in the original method. It will be generalized and made more precise in what follows. We will present new results, mostly the methods based on the Fourier transform. Sections 3.1 and 3.2 are based on [11], Sections 3.3 and 3.4 are completely new. 


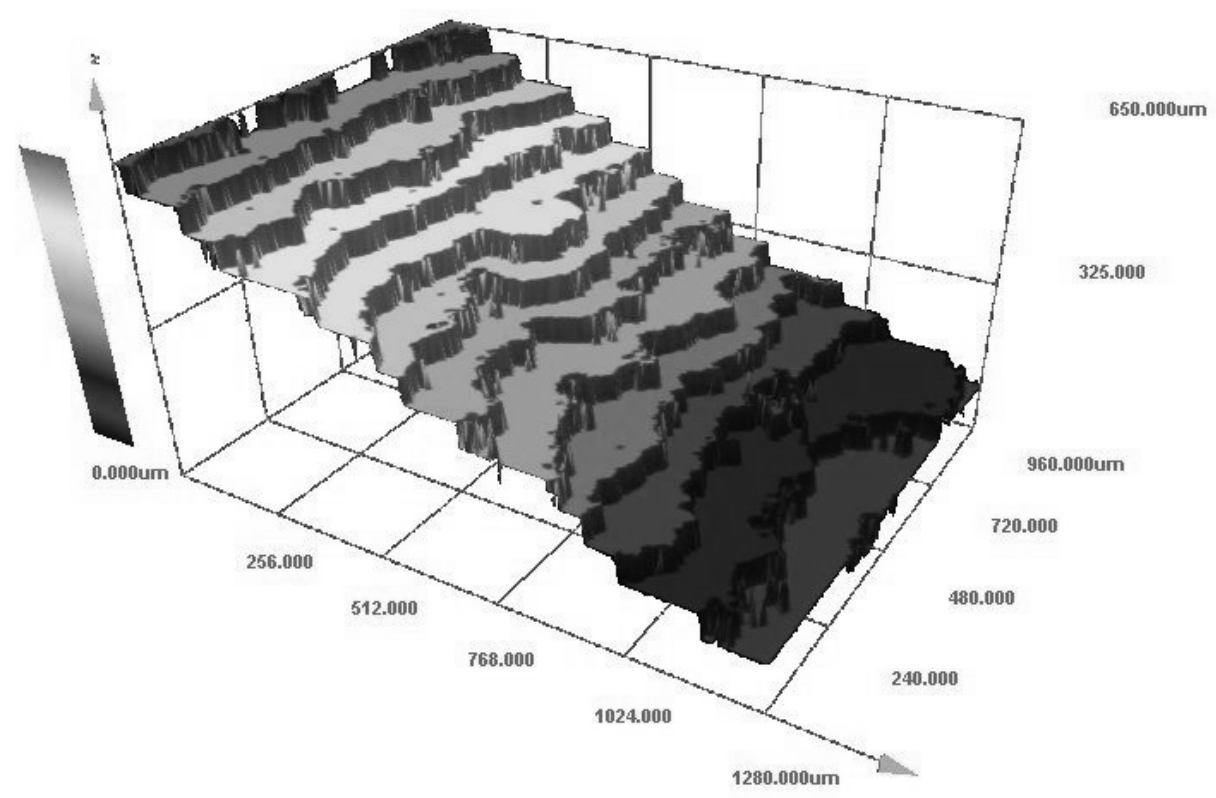

Figure 2. 3D reconstruction of the specimen from Figure 1 from a confocal microscope constructed from twelve optical cuts.

\subsection{Limits of real optical instruments}

In geometrical optics, the terms such as object space and image space are used. In what follows, we will use a relation that conforms to the postulates of geometrical optics and call it a geometrical projection. The relation (it is not a mapping in general) is implemented by direct rays that pass through the point $P$ that is to be displayed. These rays are transformed by the optical system into conjugated rays that pass through the image space and meet at a point $P^{\prime}$. However, the mapping as implemented by a real device (a microscope or a camera) does not conform exactly to the postulates of geometrical optics. A list of some of the reasons for this difference follows:

The limited width of the beam of rays. The mapping of point $P$ is carried out by a beam of rays. If we denote by $\mathcal{A}$ the set of the values assumed by the angles formed by the rays of the bunch, then $\sup \mathcal{A}=\pi$. Although the beam of rays that goes through a camera is very broad, we have always $\sup \mathcal{A}<\pi$.

The wave nature of light. Geometric optics presumes that light propagates along straight lines. However, this presumption, is valid only if light goes through a homogeneous and isotropic environment (which, in a microscope or camera, can be presumed), and further if light passes obstacles that are larger than its wavelength by orders of magnitude. This, however, is not the case of microscopes and cameras. When a microscopic preparation is observed, light is always inflected by a small obstacle - the preparation itself (see [1]). 
The optical system of the microscope as such also produces flexural phenomena, namely, on the input pupil. Thus, to give a mathematical description of an optical instrument we must also take into account the wave nature of these phenomena. This means that the geometrical projection $\mathcal{G} \subset O \times \mathcal{O}^{\prime}$ cannot be used and has to be replaced with a more general correspondence between the object space $\mathcal{O}$ and image space $\mathcal{O}^{\prime}$ and between the sharpness plane $\omega$ and the focal plane $\varphi$, too.

A relation $\mathcal{M}^{(V)} \subset \omega \times \varphi$ such that

$$
[P, Q] \in \mathcal{M}^{(V)} \Leftrightarrow Q \in \mathcal{S}_{P}^{(V)}=\left\{X \in \varphi|| X Q \mid \leq \frac{\lambda_{0}}{4 A} \wedge[P, Q] \in \mathcal{G}\right\}
$$

is called wave scanning. The set $\mathcal{S}_{P}^{(V)}$ is called the wave trace of the point $P$ in wave scanning $\mathcal{M}^{(V)}$, the number

$$
d\left(\mathcal{S}_{P}^{(V)}\right)=\sup _{X, Y \in \mathcal{S}_{P}^{(V)}}\{a \in \mathbb{R}|a=| X, Y \mid\}=\frac{\lambda_{0}}{2 A}
$$

is called its diameter, $A$ is the so-called numerical aperture of the microscope, $\lambda_{0}$ the wavelength of the light used. The wave trace of the point is illustrated in Figure 3a.

Nonplanarity of the preparation. As indicated above, it is impossible to focus the microscope so that it displays every point as a point. When observing a nonplanar preparation, further problems are encountered. As mentioned above, a sharp image can only be obtained in the sharpness plane. However, in many cases it is impossible to place the whole object in the sharpness plane, the object is only sharp in a contour line.

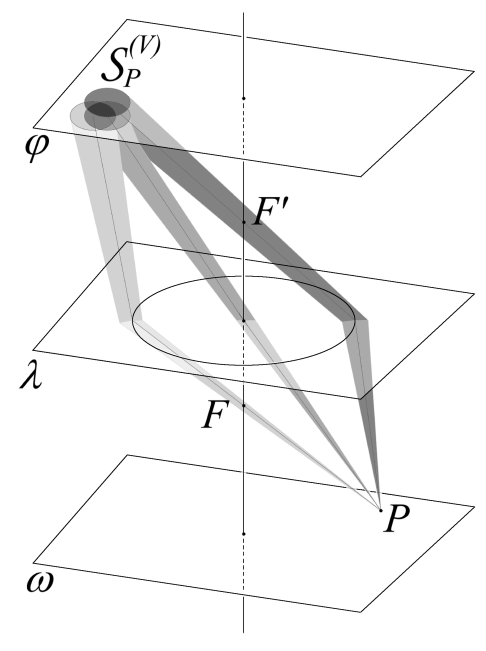

(a) Wave trace of point $P$

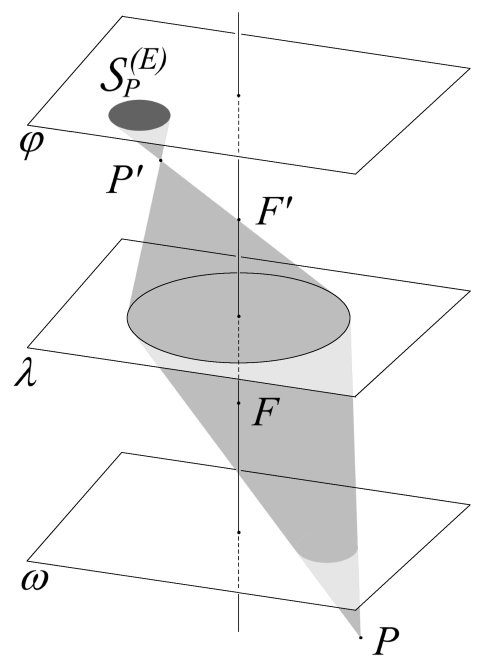

(b) Euclidean trace of point $P$

Figure 3. Wave and Euclidean trace of point $P$. 
A relation $\mathcal{M}^{(E)} \subset \omega \times \varphi$ such that

$$
[P, Q] \in \mathcal{M}^{(E)} \Longleftrightarrow Q \in \mathcal{S}_{P}^{(E)}=\{X \in \varphi \mid[P, X] \in \mathcal{G}\}
$$

is called the Euclidean scanning. The set $\mathcal{S}_{P}^{(E)}$ is called the Euclidean trace of the point $P$, the number

$$
d\left(S_{P}^{(E)}\right)=\sup \{a \in \mathbb{R}|a=| X, Y \mid\}
$$

is called its diameter.

Scanner resolution. In all the above considerations of the properties of a microscope or a camera, we assumed that all the deviations from the geometrical projection originate in the microscope during the projection itself. However, these deviations are also caused by the scanner. Assuming the straight propagation of light, let the preparation be placed flush with the microscope sharpness plane, then the geometrical projection takes every point $P$ to point $P^{\prime}$. Despite this, the point cannot be mapped to a point. A scanner is a physical object and, therefore, it cannot represent the point as a dimensionless object. According to Section 1, every point is displayed as a physical pixel with nonzero dimensions.

The image acquired by a particular instrument is the result of intricate interactions of the phenomena described above. An accurate mathematical description of these interactions would be extremely complicated. However, as will be demonstrated in what follows and briefly mentioned in Section 2.2, there are interesting possibilities of reconstructing real images.

\subsection{Multifocal image}

In the previous section, we indicated that, in a nonplanar preparation, only the contour line in which the preparation intersects the sharpness plane is displayed sharply. However, the whole $3-\mathrm{D}$ object would be unsharp in this case. The conclusions concerning the focusing contour line are based on the assumption that the sharpness plane and the focal plane are Euclidean planes.

If the point to be displayed lies outside the sharpness plane, it is displayed into its Euclidean trace, whose radius depends on the distance of the point from the plane. As the distance increases, so does the radius. However, the dependence is not strictly proportional. If it was possible to display Euclidean points in the plane, every Euclidean trace with a strictly positive diameter would cause an outof-focus image. If the plane of the scanning device is a set of physical pixels, the unsharpness is only manifested if $d\left(\mathcal{S}_{P}^{(E)}\right)>q$ where $q=\min \{x, y\}$. If $d\left(\mathcal{S}_{P}^{(E)}\right) \leq q$, we can take the picture as sharp.

Let $P$ be a point of the objective space, $\mathcal{M}^{(E)} \subset \omega \times \varphi$ an Euclidean scanning, $\mathcal{P}$ the physical plane of the focal plane $\varphi, x, y$ the dimension of its physical pixels ( $x \neq y$ in the general case of rectangle pixels), $d\left(\mathcal{S}_{P}^{(E)}\right)$ the diameter of the Euclidean trace of point $P$. We call the set

$$
\mathcal{Z}(\mathcal{O})=\left\{P \in \mathcal{O} \mid d\left(S_{P}^{(E)}\right)<q, \quad q=\min \{x, y\}\right\}
$$


an open zone of sharpness. Its image $\mathcal{G}(\mathcal{Z})$ in the geometrical projection $\mathcal{G}: \mathcal{O} \rightarrow$ $\mathcal{O}^{\prime}$ is called an optical cut.

The conclusions concerning the focusing contour line are based on the assumption that the sharpness level and the focal plane are Euclidean planes, but this assumption is not valid. If the point to be displayed lies outside the sharpness plane, it is displayed into its Euclidean trace, whose radius depends on the distance of the point from the plane. As the distance increases, so does the radius. However, the dependence is not strictly proportional. If it was possible to display Euclidean points in the plane, every Euclidean trace with strictly positive diameter would cause an out-of-focus image.

It is evident that the result of a scanning depends not only on the object observed, but also on the instrument focusing. The same preparation may be observed with different focusings, that is, with different settings of the focal planes. If the number of these settings is $n$ (in general), we will obtain $n$ different scannings: $n$ different zones of sharpness $\mathcal{Z}_{k}, k=1,2, \ldots, n$, and $n$ different optical cuts $\mathcal{G}_{k}\left(\mathcal{Z}_{k}\right), k=1,2, \ldots, n$ of object $\mathcal{O}$. It is evident that it is only possible to obtain a sharp image by a single scanning if the zone of sharpness is wider then the $3 \mathrm{D}$ object height. If, however, it is narrower than the preparation height, a part of the preparation is always out of focus. To construct a sharp picture in such a case, a multifocal image is required. It is a sequence $\left\{\mathcal{O}_{k}^{\prime}\right\}_{k=1}^{n}$ of images of object $\mathcal{O}$ in the geometrical projections $\mathcal{G}_{k}$ whose zones of sharpness $\mathcal{Z}_{k}$ cover the whole object $\mathcal{O}$, i.e.,

$$
\mathcal{O} \subseteq \bigcup_{k=1}^{n} \mathcal{Z}_{k}(\mathcal{O})
$$

\section{3. $2 \mathrm{D}$ processing}

The two-dimensional (2D) processing of an $n$-focal image involves constituting a new image so that this new image consists of the optical cuts of the multifocal image $\left\{\mathcal{O}_{k}^{\prime}\right\}_{k=1}^{n}$. In the next step, we will set criteria for assigning each pixel in the images $\left\{\mathcal{O}_{k}^{\prime}\right\}_{k=1}^{n}$ to an optical cut $\mathcal{G}_{k}\left(\mathcal{Z}_{k}\right)$.

Human eyes can see a part of the image sharply when the high spatial frequencies in the sense of the Fourier transform are present, see $[2,3]$. Therefore, a neighborhood of each pixel $[i, j]$ of the multifocal image $\left\{\mathcal{O}_{k}^{\prime}\right\}_{k=1}^{n}$ is transformed by the Fourier transform. A sum through a suitable annulus with the center in the center of the amplitude spectrum (at coordinate $[0,0]$ ) is a suitable criterion for image contrast and sharpness diagnostics. We use the characteristic

$$
C_{P(i, j)}^{(k)}=\frac{1}{m\left(A_{i j}\right)} \sum_{[r, s] \in A_{i j}} f^{(k)}(r, s),
$$

where $f^{(k)}(r, s)$ is the value of the pixel $[r, s]$ in image $\mathcal{O}_{k}^{\prime}$ and $m\left(A_{i j}\right)$ is the number of pixels in the annulus $A_{i j}$ (shown in gray in Figure 4).

Higher values of $C_{i j}^{(k)}$ mean higher amplitudes on higher spatial frequencies, which indicates higher contrast of small details in the studied neighborhood and thus also a better focusing. Therefore, the expression (3.1) is used as a focusing criterion. Figure 5 shows the sharpness zones detected by Equation (3.1) for 

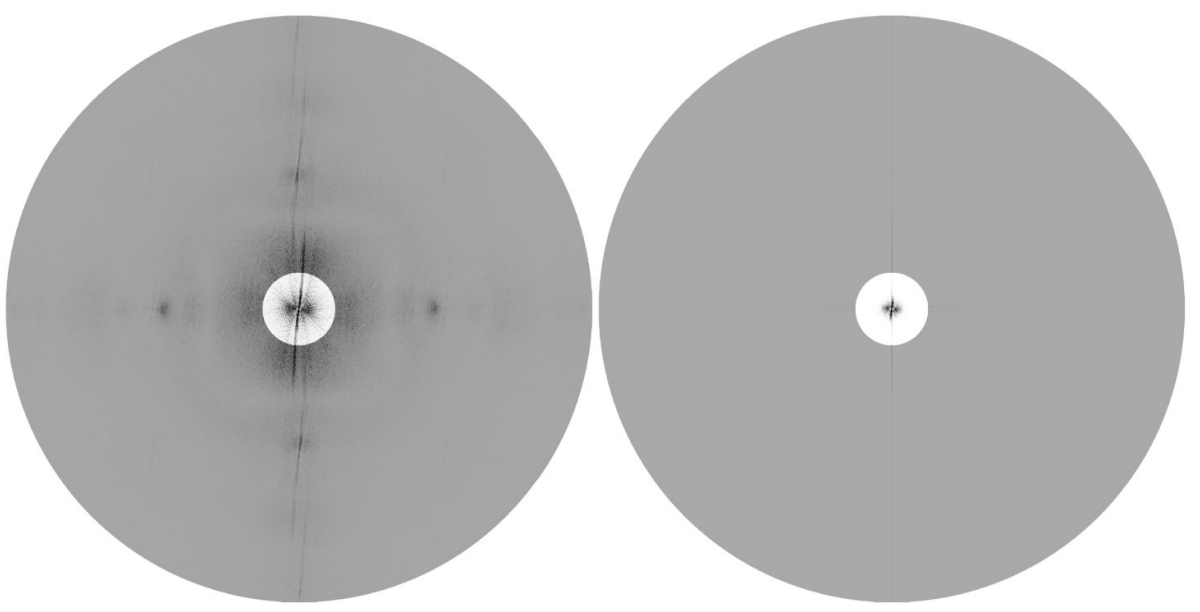

Figure 4. Amplitude spectra of the same neighborhood of pixel $[i, j]$ in different images $\mathcal{O}_{k}^{\prime}$ of multifocal image $\left\{\mathcal{O}_{k}^{\prime}\right\}_{k=1}^{n}$. This pixel is better focused in the left image $O_{k}^{\prime}$. Amplitude spectra are in negative, i.e., the highest values of the amplitude spectrum are black.

a multifocal image composed of twelve images (the second, the fourth, the eighth, and the tenth are shown in Figure 1). This image $\mathcal{O}_{0}^{\prime}$ is called the compositional image and it is possible to define it as

$$
\mathcal{O}_{0}^{\prime}: M \rightarrow \mathbb{N}, \text { where } \mathcal{O}_{0}^{\prime}(i, j)=k \Longleftrightarrow C_{i, j}^{(k)}=\max _{l=1,2, \ldots n}\left\{C_{i, j}^{(p)}\right\} .
$$

Note that $[i, j] \in M=\{0,1, \ldots, w-1\} \times\{0,1, \ldots, h-1\}$ as in Section 1. A focused $2 \mathrm{D}$ image $\mathcal{F}$ is subsequently constructed from maximally focused pixels

$$
\mathcal{F}: M \rightarrow R \subset \mathbb{R} \text {, where } \mathcal{F}(i, j)=\mathcal{O}_{k}^{\prime}(i, j) \Longleftrightarrow C_{i, j}^{(k)}=\max _{l=1,2, \ldots, n}\left\{C_{i, j}^{(p)}\right\} .
$$

(see Figure 6).

\subsection{D processing}

In the previous section, we used the focusing criterion only as a qualitative characteristic - based on the maximum, we assigned an optical cut to each pixel. In the $3 \mathrm{D}$ reconstruction, we can assign the same height to all pixels that belong to one optical cut. In this way, we can obtain $n$ levels from $n$ focal images (see [9] and [10] for example). This method is called the Method of Constant High Cuts.

Figure 7 shows a 3D reconstruction of data whose sample is in Figure 1. The difference between this method and Greenberg and Boyde is that Fourier transform (Section 3.3) was used for detection of optical cuts.

Thanks to the focusing criterion described above, we can use even classical instruments (a standard microscope or a CCD camera) for much higher quality $3 \mathrm{D}$ reconstruction.

The radius of the Euclidean trace and wave trace of the point depend on the distance of this point from the sharpness plane. The radius of these traces increases with the distance from the sharpness plane. The focusing criteria parameters are 


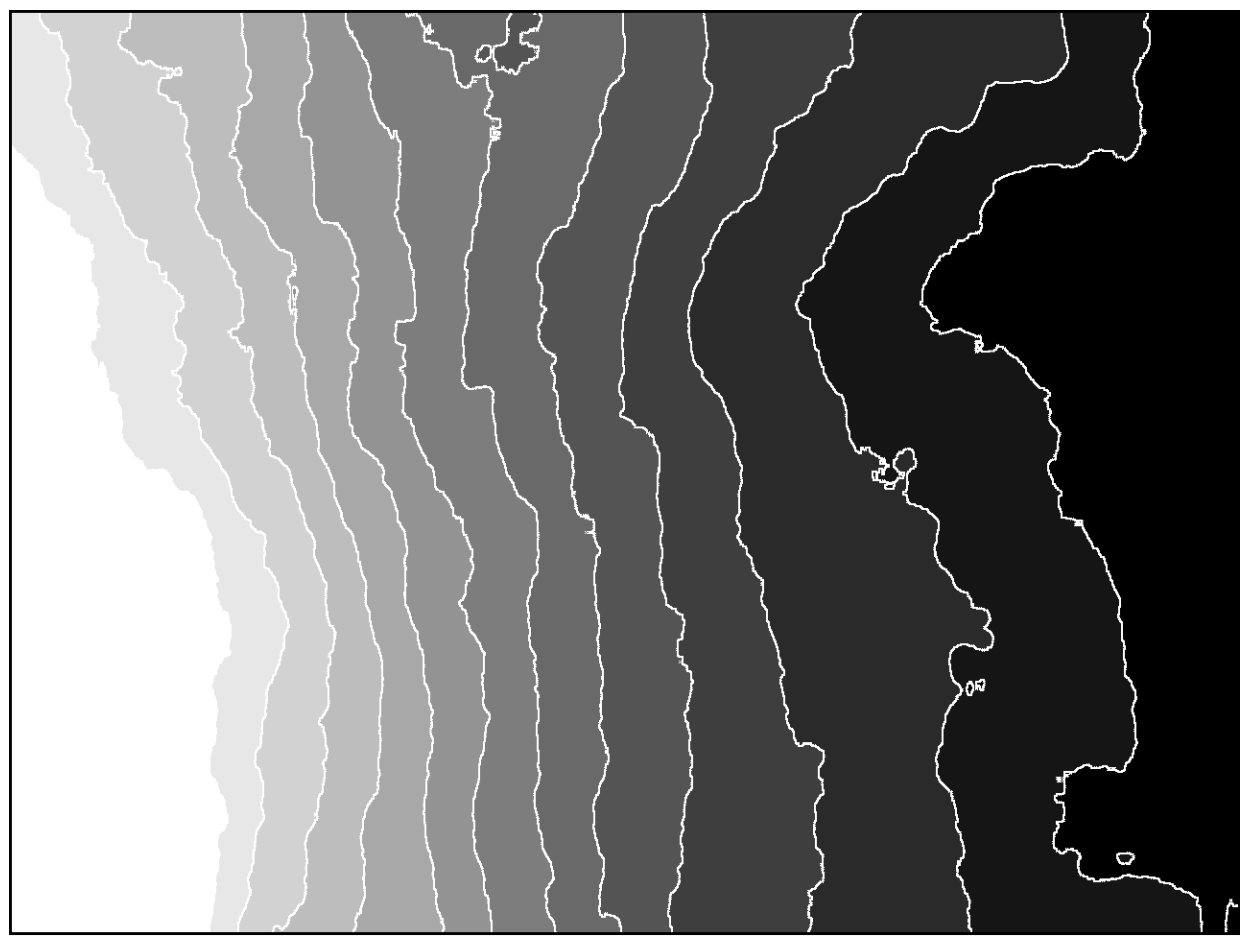

Figure 5. Compositional image $\mathcal{O}_{0}^{\prime}$. Optical cuts detected by expression (3.1). Different colors show different images of the multifocal image as the sharpest images in the image set.

changed accordingly. Analyzing the focusing criteria parameters for all images in the multifocal image we can determine this distance for each pixel more precisely than in Section 2.2. In this way, it is possible to obtain a 3-D object profile very easily.

We assume that the value $C_{i j}^{(k)}$ of the focusing criterion in the particular image $\mathcal{O}_{k}^{\prime}$ is inversely proportional to the radius $d\left(\mathcal{S}_{P}^{(E)}\right)$ of the Euclidean trace $\mathcal{S}_{P}^{(E)}$ of the point $P$, which is observed in physical pixel $P(i, j)$ in the Euclidean scanning $\mathcal{M}^{(E)}$.

Let us consider a sequence $\mathcal{M}_{k}^{(E)}, k=1,2, \ldots, n$, of Euclidean scannings that formed a multifocal image $\left\{\mathcal{O}_{k}^{\prime}\right\}_{k=1}^{n}$. Optical cuts $\mathcal{M}_{k}^{(E)}\left(\mathcal{Z}_{k}\right)$ of particular images $\mathcal{O}_{k}^{\prime}$ are images of zones of sharpness $\mathcal{Z}_{k}$ with planes of sharpness $\omega_{k}, k=1,2, \ldots, n$. Let $\omega_{r}$ be a plane of sharpness and let the points $P_{1}, P_{2}$ lie in the zone of sharpness $\mathcal{Z}_{r}$ according to Figure 8 .

Next, consider the plane of sharpness $\omega_{s}$ of the zone $\mathcal{Z}_{s}, s \neq r$. For the distances $h^{(s)}\left(P_{1}\right), h^{(s)}\left(P_{2}\right)$ of points $P_{1}, P_{2} \in \mathcal{Z}_{r}$ from plane $\omega_{s}$ it holds $h^{(s)}\left(P_{1}\right)<$ $h^{(s)}\left(P_{2}\right)$, which means that $C_{P_{1}}^{(s)}>C_{P_{2}}^{(s)}$. Let us denote $C_{\mathrm{sup}}^{(s)}=\sup _{P \in \mathcal{Z}_{r}}\left\{C_{P}^{(s)}\right\}$, 


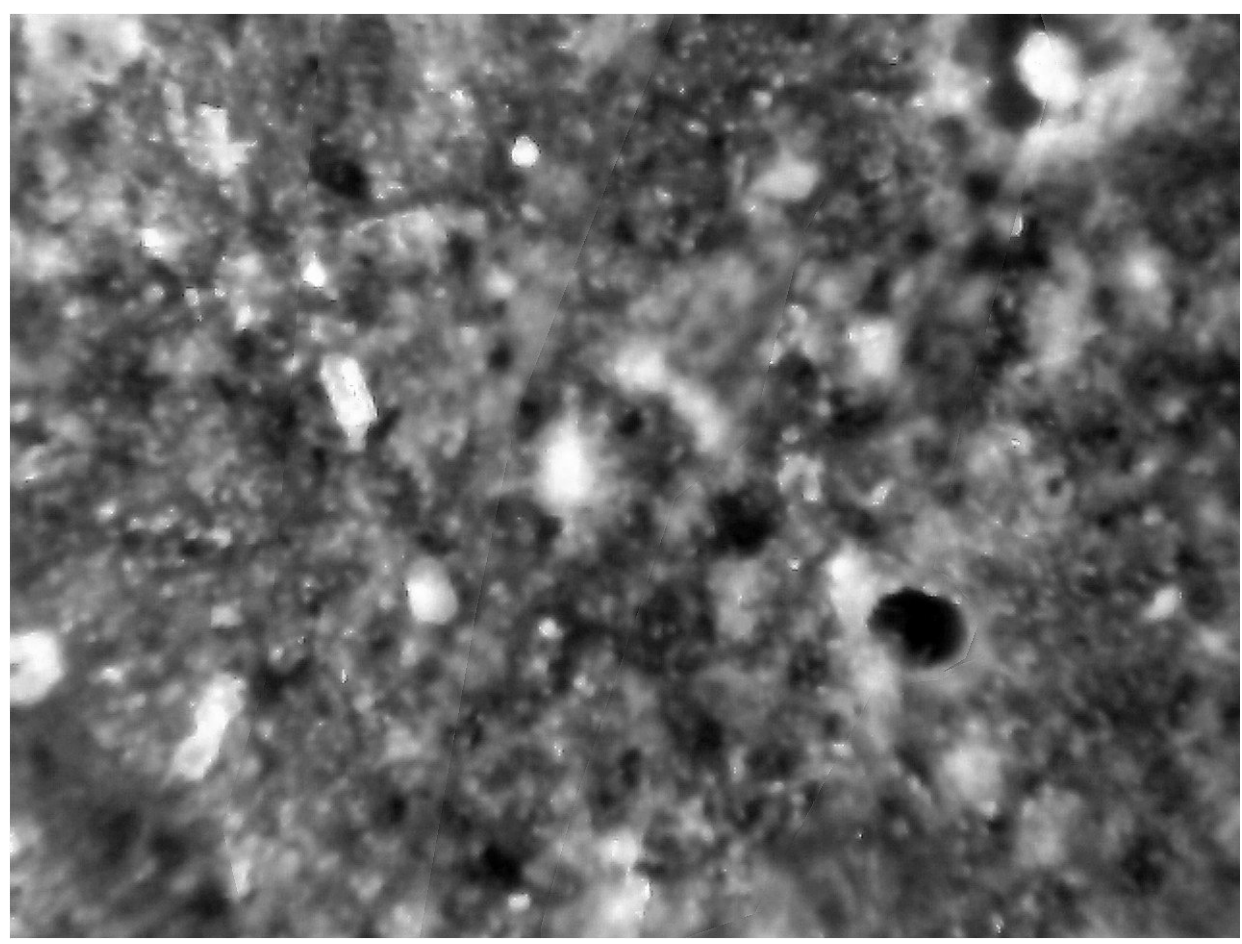

Figure 6. Focused image composed from a multifocal image $\left\{\mathcal{O}_{k}^{\prime}\right\}_{k=1}^{12}$ (four images of them are shown in Figure 1). Optical cuts were detected by expression (3.1), image was composed by expression (3.2).

$C_{\text {inf }}^{(s)}=\inf _{P \in \mathcal{Z}_{r}}\left\{C_{P}^{(s)}\right\}$, which means that

$$
\begin{aligned}
& C_{P}^{(s)}=C_{\text {sup }}^{(s)} \Longleftrightarrow h^{(s)}(P)=h_{\text {inf }}^{(s)}(P)=\inf _{P \mathcal{Z}_{r}}\left\{h^{(s)}(P)\right\}, \\
& C_{P}^{(s)}=C_{\text {inf }}^{(s)} \Longleftrightarrow h^{(s)}(P)=h_{\text {sup }}^{(s)}(P)=\sup _{P \in \mathcal{Z}_{r}}\left\{h^{(s)}(P)\right\} .
\end{aligned}
$$

We assume that the value of $C_{P}^{(s)}$ is inversely proportional to the distance $h^{(s)}(P)$ of point $P \in \mathcal{Z}_{r}$ from plane $\omega_{s}$ for every $P \in \mathcal{Z}_{r}$ and, therefore,

Since

$$
\frac{h^{(s)}(P)-h_{\mathrm{inf}}^{(s)}}{h_{\mathrm{sup}}^{(s)}-h_{\mathrm{inf}}^{(s)}}=\frac{C_{\mathrm{sup}}^{(s)}-C_{\mathrm{inf}}^{(s)}}{C_{P}^{(s)}-C_{\mathrm{inf}}^{(s)}} .
$$

it holds that

$$
h_{\mathrm{inf}}^{(s)}=\left(|r-s|-\frac{1}{2}\right) \cdot \Delta z, \quad h_{\mathrm{sup}}^{(s)}=\left(|r-s|+\frac{1}{2}\right) \cdot \Delta z,
$$

$$
h^{(s)}(P)=\frac{C_{\mathrm{sup}}^{(s)}-C_{\mathrm{inf}}^{(s)}}{C_{P}^{(s)}-C_{\mathrm{inf}}^{(s)}}+\left(|r-s|-\frac{1}{2}\right) \cdot \Delta z,
$$

where $\Delta z$ is the height of the zone of sharpness. This method enables us to calculate the height of each point $P$ with a precision higher than $\frac{\Delta z}{2}$. Figure 9 


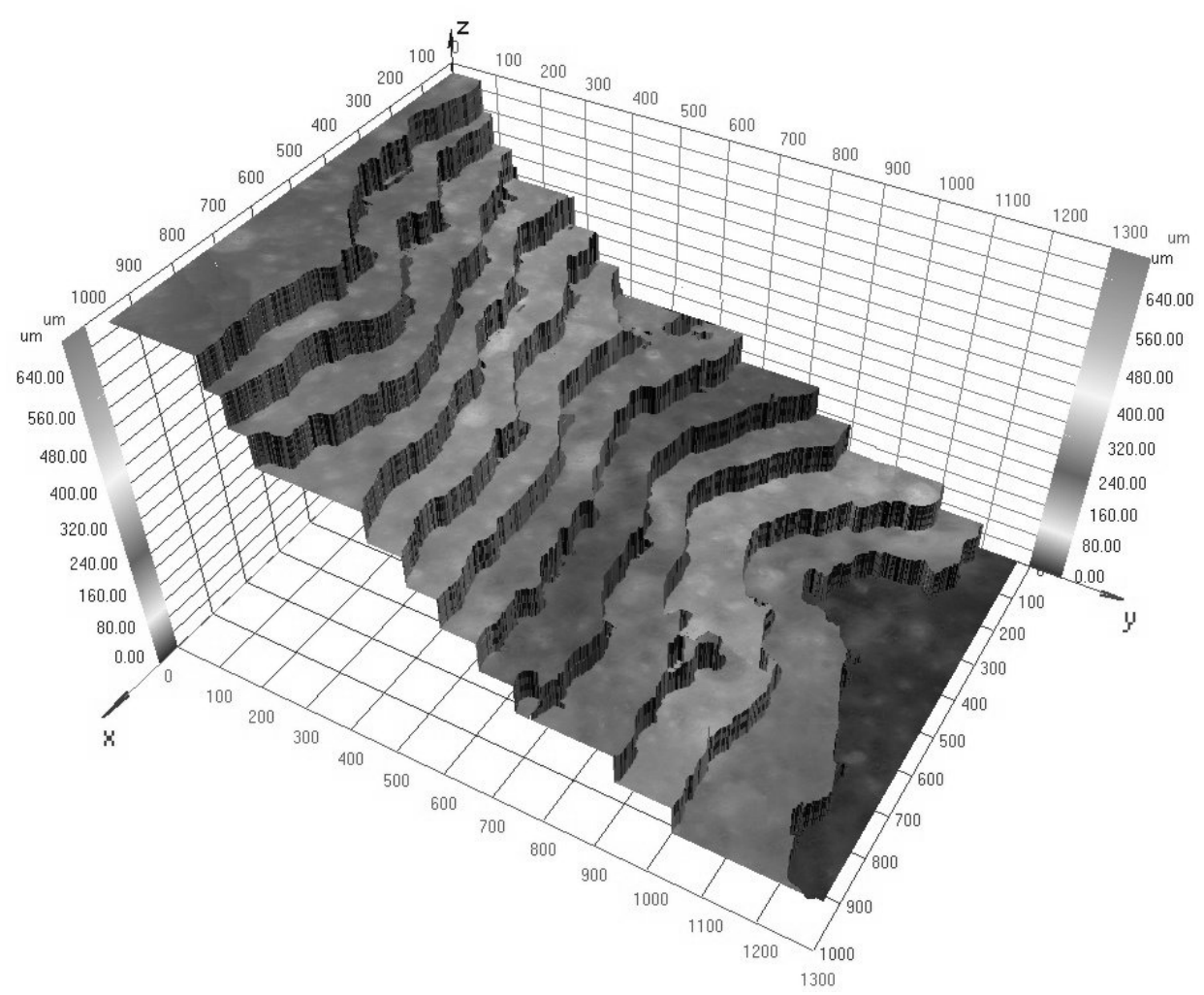

Figure 7. 3D reconstruction of a preparation by means of the Method of Constant High Cuts. A multifocal image with $n=12$ (see Figure 1) was obtained by a CCD camera, optical cuts identified with the method described in Section 3.3.

shows a 3D reconstruction of the same object from the same number of cuts $(n=12)$ as in Figure 2 and 7 . The quality of this reconstruction is similar to that of the reconstruction of the same object as obtained with a confocal microscope using the Method of Constant High Cuts for $n=150$ (Figure 10).

\section{Conclusion}

Reconstructions of three-dimensional object surfaces are an important task in many branches of research. Even though the standard method of imaging object surfaces is the use of confocal microscopes, there are methods that use optical microscopes and sophisticated post-processing methods for $2 \mathrm{D}$ and $3 \mathrm{D}$ reconstruction of objects. This enables us to obtain results similar to this from a confocal microscope with equipment by orders less expensive. This paper summarizes these methods introducing more precise methods both for $2 \mathrm{D}$ and $3 \mathrm{D}$ reconstruction based on the Fourier transform. In this way, we can obtain results similar to images from confocal microscopes with affordable and much less expensive instruments. 


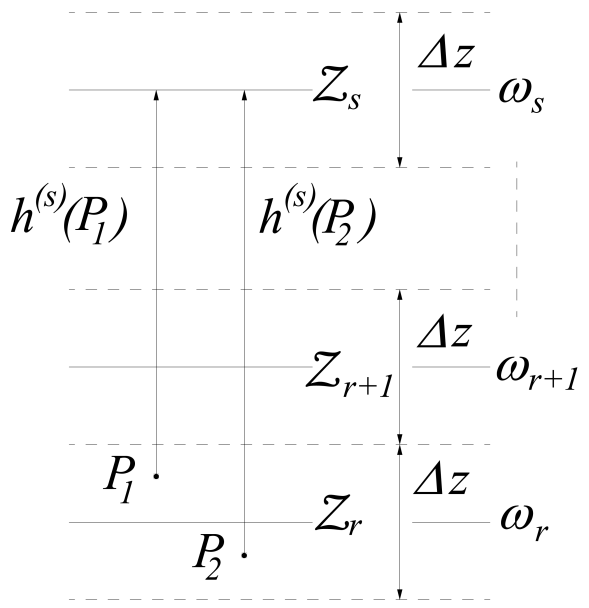

Figure 8. Illustration to computing the distance $h^{(s)}(P)$ of point $P$ from plane $\omega_{s}$.

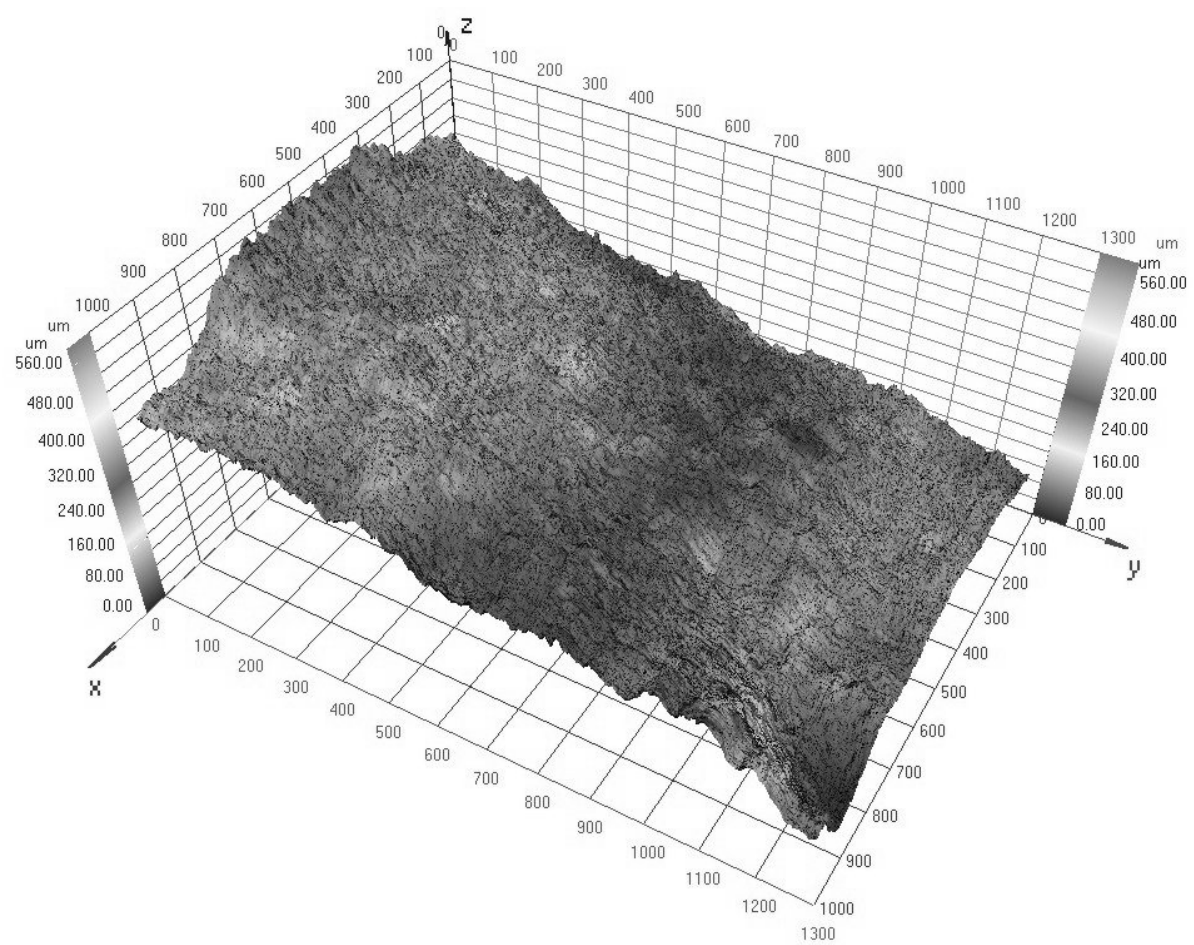

Figure 9. 3D reconstruction of the multifocal image with $n=12$ by means of Equation (3.4).

Acknowledgement. The authors thank prof. Tomáš Ficker from the Faculty of Civil Engineering of Brno University of Technology for the provided data. 


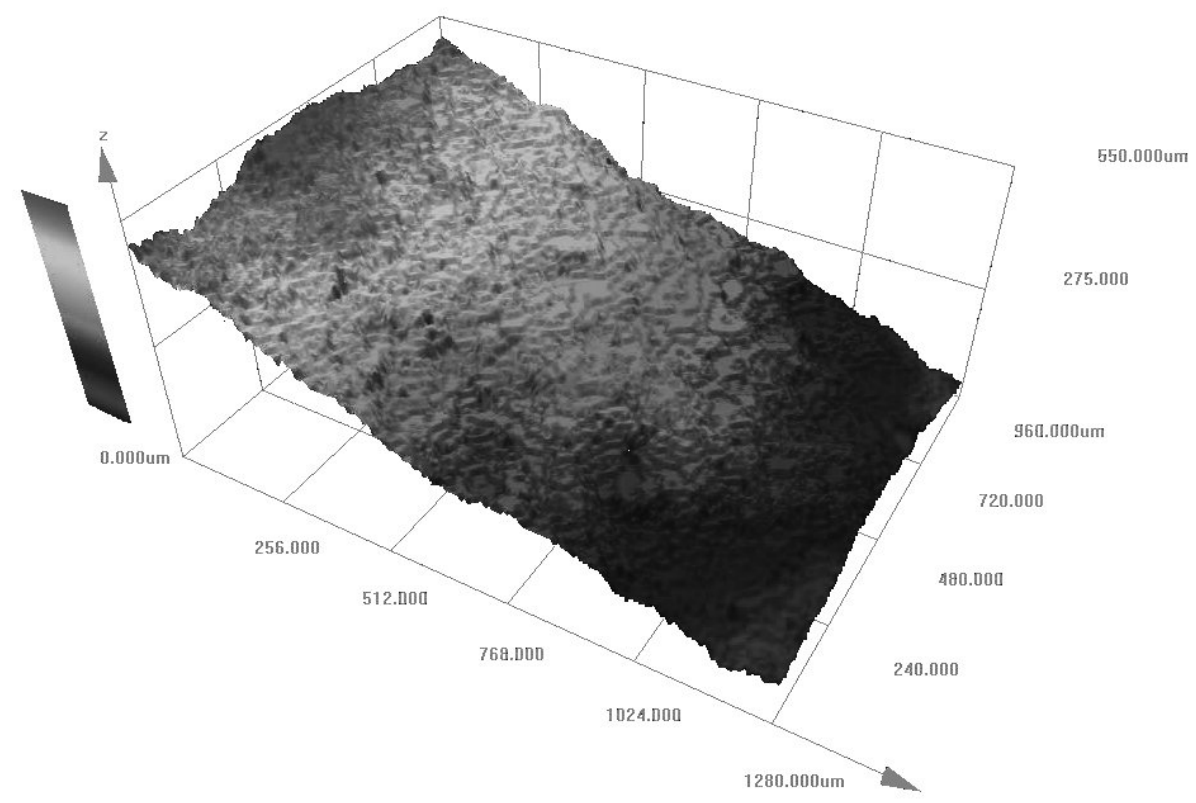

Figure 10. 3D reconstruction from a confocal microscope obtained with the Method of Constant High Cuts for $n=150$.

\section{REFERENCES}

[1] K. B. Benson, Television Engineering Handbook, 2nd ed., McGraw-Hill, New York, 1986.

[2] M. Druckmüller and P. Heriban, Scientific Image Analyser DIPS 5.0, SOFO Brno, 1996.

[3] M. Druckmüller and P. Štarha, ACC 4.0 Scientific object structure and object analyser, SOFO Brno, 2000.

[4] T. Ficker et al., Fracture surfaces of porous materials, Europhys. Lett. 80 (2007), 1600-1604.

[5] T. Ficker et al., Roughness of fracture surfaces and compressive strength of hydrated cement pastes, Cement Concrete Res. 40 (2010), 947-955.

[6] T. Ficker and D. Martišek, Digital fracture surfaces and their roughness analysis: Applications to cement-based materials, Cement Concrete Res. 42 (2012), 827-833.

[7] B. Geldziler, C. W. Hewitt, E. J. Doolin and E. Pro, Depth of field of mycology specimens using high definition 3-D microscopy employing multiple oblique illumination (abstr.), American Society for Microbiology 270 (1998).

[8] B. Geldziler, R. Ramsamooj, E. Catalano, E. J. Doolin and C. W. Hewitt, Novel high definition 3-D microscopy for evaluating cytopathology and pap smear specimens: 3-D digital image reconstruction and analysis of cell clusters (abstr.), Acta Cytol. 42 (1998), 1299.

[9] G. Greenberg, Direct 3-D imaging using a multiple oblique microscope, Scanning 16 (1994), 248-249.

[10] G. Greenberg and A. Boyde, Direct-view 3-D microscopy using conventional lenses, European Microscopy and Analysis 22, (1997), 7-9.

[11] D. Martišek, The 2-D and 3-D processing of images provided by conventional microscopes, Scanning 24 (2002), 284-296. 
Dalibor Martišek, Institute of Mathematics, Faculty of Mechanical Engineering, Brno University of Technology, Technická 2, 61669 Brno, Czech Republic e-mail: martisek@fme.vutbr.cz

Hana Druckmüllerová, Institute of Mathematics, Faculty of Mechanical Engineering, Brno University of Technology, Technická 2, 61669 Brno, Czech Republic

e-mail: ydruck00@stud.fme.vutbr.cz 